\title{
Uma nova métrica para protocolos de roteamento em redes em malha sem fio
}

\author{
Dalbert Mascarenhas ${ }^{1}$, Marcelo Rubinstein ${ }^{1,2}$, Alexandre Sztajnberg ${ }^{1,3}$ \\ ${ }^{1}$ Programa de Pós-Graduação em Engenharia Eletrônica (PEL) \\ Universidade do Estado do Rio de Janeiro (UERJ) \\ ${ }^{2}$ Departamento de Eletrônica e Telecomunicações / FEN \\ ${ }^{3}$ Departamento de Informática e Ciência da Computação / IME \\ \{dalbert, rubi, alexszt\}@uerj.br
}

\begin{abstract}
Resumo - Este artigo propõe uma nova métrica, AP (Alternative Path), a ser utilizada para o cálculo de rotas em protocolos de roteamento em redes em malha sem fio. Esta métrica leva em consideração a interferência causada por nós vizinhos na escolha de uma rota para um destino. $O$ desempenho da métrica AP é avaliado e comparado com o da métrica ETX (Expected Transmission Count). As simulações realizadas mostram que a métrica AP pode propiciar desempenho superior à rede.
\end{abstract}

Palavras-chave - redes em malha, métricas de roteamento, protocolos de roteamento.

Abstract - This paper proposes a new metric, AP (Alternative Path), to be used in the calculation of routes in mesh network routing protocols. This new metric is concerned with the interference caused by neighbor nodes when choosing a route for a destination. The performance of the AP metric is evaluated and compared to the ETX (Expected Transmission Count) metric. Simulations show that AP can provide superior performance to the network.

Key-words - mesh networks, routing metrics, routing protocols.

\section{INTRODUÇ̃̃O}

Redes em malha sem fio (wireless mesh networks) têm atraído a atenção da indústria e de pesquisadores [1] por seu custo reduzido para uma cobertura geográfica relativamente grande, se comparado a outras redes, e também devido à facilidade de implantação em áreas onde o uso de infraestrutura cabeada seria inviável. Universidades têm sido pioneiras no uso de redes em malha para interligar prédios de seus campi e prover acesso à Internet para seus alunos e colaboradores [2], [3], [4]. Alguns projetos urbanos também começam a utilizar redes em malha para prover conexão entre pontos que apresentam grandes distâncias geográficas e acesso à Internet para a comunidade [5].

Uma das principais características das redes em malha está no uso de roteadores sem fio, geralmente fixos, com maior poder de processamento em relação aos roteadores móveis, que em geral apresentam suprimento de energia limitado. Estes roteadores têm duas funções: (i) encaminhar tráfego para os outros roteadores que fazem parte da rota de destino e (ii) receber e encaminhar tráfego para os nós clientes, que podem estar conectados através de uma estrutura cabeada ou usar a comunicação sem fio.

A auto-organização e a auto-configuração devem ser características presentes nas redes em malha, de forma que a manutenção das conexões entre os roteadores seja automática [6]. Tais características facilitam a inclusão de novos nós roteadores na rede, ampliando sua cobertura. Além disso, tornam as redes tolerantes a falhas devido aos múltiplos caminhos criados entre nós roteadores e nós clientes.

Atualmente uma parte significativa dos protocolos de roteamento adotados em redes em malha são adaptações de protocolos de roteamento de redes ad hoc. Entretanto, a utilização de protocolos de roteamento ad hoc pode provocar queda de desempenho nas redes em malha, principalmente porque os protocolos de roteamento ad hoc foram desenvolvidos para dar suporte a cenários em que os nós são móveis.

O comportamento e o desempenho de uma rede em malha recaem principalmente na combinação de métricas utilizadas no protocolo de roteamento. Grande parte das pesquisas envolvendo protocolos de roteamento e métricas para redes em malha têm dado importância à diminuição do atraso e ao aumento da vazão[6].

A importância das métricas de roteamento está vinculada ao que se espera de determinadas redes em malha. Algumas métricas levam em conta a taxa de perda de pacotes, outras utilizam múltiplos canais para proporcionar um melhor aproveitamento do meio físico, outras também usam como medida a largura de banda. Existem também as métricas que levam em consideração a quantidade de nós competindo pelo mesmo meio físico e a interferência causada pelos mesmos em seus vizinhos [7].

Este trabalho propõe uma métrica que em adição à informação da taxa de perdas de pacotes nos enlaces, também utiliza a informação do número de vizinhos dos nós. A idéia é dar preferência à utilização de rotas com menor probabilidade de interferências. Dessa forma, rotas alternativas são escolhidas com o objetivo de oferecer melhor desempenho à rede. Esta métrica será chamada de Alternative Path (AP). 
O artigo está organizado da seguinte forma. Na Seção II são apresentados os trabalhos relacionados, destacando os principais protocolos de roteamento e métricas. A métrica proposta é apresentada na Seção III. Na Seção IV apresentamos simulações com a métrica AP e avaliamos seu desempenho. Por fim, a Seção V apresenta a conclusão do trabalho.

\section{TRABALHOS RELACIONADOS}

\section{A. Protocolos de roteamento}

Os protocolos de roteamento para redes em malha se dividem em reativos e proativos. Os reativos fazem a descoberta de rotas sob demanda, apenas quando um nó solicita um caminho até o seu nó destino. A abordagem reativa é adequada para as redes ad hoc principalmente devido à alta mobilidade dos nós. Criar as rotas apenas quando se deseja enviar as informações faz com que se evite o desperdício de energia. Por outro lado, os protocolos proativos fazem a atualização de suas tabelas de rotas periodicamente. Essa característica faz com que cada nó tenha uma visão global da topologia da rede. Como nas redes em malha o limite de energia e a mobilidade não são fatores limitantes, a atualização periódica das rotas abre espaço para o envio de outras informações que viabilizem a utilização de métricas que levam em conta a qualidade dos enlaces.

Como mencionado, alguns dos protocolos de roteamento das redes ad hoc foram empregados em redes em malha [8], ainda que impedindo o aproveitamento de alguns recursos que estão presentes nas redes em malha, como o maior poder de processamento dos nós fixos e o maior suprimento de energia. São exemplos de protocolos ad hoc usados em redes em malha, o AODV [9] e o DSR [10]. Tanto o AODV quanto o DSR são protocolos reativos.

Determinados protocolos de roteamento utilizados em redes em malha atualmente como o LQSR [11] e o SrcRR [12], fazem suas descobertas de rotas de forma similar aos protocolos ad hoc reativos. O LQSR utiliza tanto o roteamento reativo como o proativo de forma com que se tenha um equilíbrio entre as trocas de mensagens topológicas e a visão global da rede. O SrcRR faz uso do recurso reativo para atualizar as rotas usadas, diminuindo a quantidade de mensagens de controle e reduzindo o mapa topológico de cada nó. O MR-LQSR [13] foi desenvolvido a partir do LQSR para trabalhar com múltiplos canais, técnica muito utilizada para evitar a sobrecarga no mesmo canal.

Segundo Campista et al. [14], o grande número de mensagens de controle fazendo anúncios de estado de enlace pode comprometer a escalabilidade da rede. Com base nisso, alguns protocolos proativos evitam inundar toda a rede com suas mensagens de controle. Em alguns casos o protocolo de roteamento não propaga as mensagens de controle para todos os nós com a mesma freqüência. Existem também os protocolos de roteamento que selecionam determinados nós como encarregados de repassar as mensagens de controle, dessa forma evitando a sobrecarga de mensagens de controle na rede.
Para enviar mensagens de controle somente para os nós que tenham uma relevância no cálculo da rota, o protocolo de roteamento Fisheye [15] ajusta o TTL (Time-To-Live) das mensagens de controle de forma que se alcance o número de nós desejável. Diminuindo o TTL, reduz-se também o número de nós inundados pelas mensagens de controle. Já o MMRP [16] estipula um tempo de vida para os estados de enlace. Ao encaminhar uma mensagem de controle, subtrai-se do tempo de vida do estado de enlace o tempo estimado para encaminhar a mensagem de controle. Dessa forma, ao expirar o tempo de vida do estado de enlace, o mesmo é descartado evitando a propagação para todos os nós da rede.

Entre os protocolos que selecionam determinados nós para propagarem as mensagens de controle, o OLSR [17] se destaca. No OLSR cada nó escolhe um conjunto de nós, chamados de MPR (MultiPoints Relays), que terão a tarefa de encaminhar as mensagens de controle. Fatores como a simetria do enlace e a quantidade de vizinhos de dois saltos atingidos são considerados na escolha de um MPR. A utilização de um conjunto de MPRs limita o número de mensagens de controle propagadas e favorece o desempenho da rede ao evitar as tempestades de broadcasts comuns em protocolos de roteamento, ao tentarem criar e manter o estado de enlace.

\section{B. Métricas}

A escolha de métricas, o peso de cada métrica e as heurísticas utilizadas para a construção das tabelas de rotas em cada nó têm influência direta no comportamento e desempenho da rede. O objetivo é que os nós consigam utilizar os melhores caminhos, evitando perdas de pacotes, loops de roteamento e instabilidade na rede.

O número de saltos é uma métrica que seleciona as rotas com menor número de nós intermediários [18]. Essa métrica é utilizada em protocolos ad hoc devido à mobilidade dos nós. Como os nós estão em movimento, quanto maior o número de nós para se atingir um nó destino, maior é a probabilidade de haver falhas nas rotas. Porém em redes em malha a utilização da métrica de número de saltos pode levar a um desempenho ruim da rede, pois negligencia a qualidade dos enlaces. Em função disso, novas métricas que levam em consideração as variações do meio físico surgiram.

A métrica ETX (Expected Transmission Count) pode ser definida como o número esperado de transmissões necessárias para a entrega de um pacote através de um determinado enlace [19]. Essa métrica calcula o peso de uma determinada rota através do somatório de todos os ETXs dos enlaces de um caminho. Isso garante o objetivo proposto pela métrica de escolher rotas que diminuam o número total de retransmissões no nível de enlace, ao longo do caminho.

Os resultados das comparações entre as métricas ETX e a métrica número de saltos demonstram que a ETX melhora o desempenho da rede ao se escolher rotas com menor probabilidade de perdas de pacotes [20]. Porém, a métrica ETX pode não refletir com exatidão a probabilidade destas perdas, pois a mesma utiliza pacotes de broadcast para inferir as probabilidades de transmissão e estes pacotes possuem um tamanho reduzido. A métrica pode fazer uma estimativa menos realista do que se estivesse usando pacotes maiores para inferir 
a probabilidade de transmissão. Além disso, estes pacotes são enviados em uma taxa básica de transmissão (taxa física).

Como alternativa à métrica ETX existe a métrica ML [2] que utiliza uma abordagem multiplicativa no custo de cada enlace. A ML busca encontrar caminhos mais seguros em relação a perdas de pacotes mesmo que isso leve a escolha de rotas com mais saltos. Segundo Passos et al. [2], a métrica ML apresenta uma maior estabilidade em suas rotas quando comparada com a ETX.

Uma versão melhorada da ETX é a métrica ETT (Expected Transmission Time) [3] que utiliza em seus cálculos de melhor rota as diferentes taxas de transmissão dos enlaces. Assim o cálculo do ETT pondera o ETX de um enlace, multiplicando o mesmo pelo tamanho do pacote dividido pela taxa de transmissão do enlace. Utilizando a taxa de transmissão do enlace, a métrica pode refletir o impacto das perdas com taxas físicas mais altas de pacotes de dados. Devido à possibilidade do aumento do tamanho do pacote, a métrica pode refletir melhor as taxas de erros e perdas de pacotes de dados.

Para tentar evitar a interferência criada por nós roteadores em um mesmo caminho foi criada a métrica WCETT [13]. Sendo baseada na métrica ETT, a WCETT utiliza múltiplos canais para tentar reduzir a interferência causada por nós em um mesmo caminho que utilizem o mesmo canal. Essa interferência também é conhecida como intrafluxo. Apesar de utilizar as probabilidades de ocorrência de interferência intrafluxo em uma rota, a WCETT não considera os efeitos da interferência interfluxo. Esse efeito é causado pela interferência de nós vizinhos que transmitem fluxos concorrentes. A interferência do tipo interfluxo pode ser observada principalmente em redes onde existe uma grande quantidade de nós por área [21]. Outro problema da métrica WCETT está na dificuldade de ser utilizada em algoritmos eficientes [7].

As métricas MIC (Metric of Interference and Channelswitching) [22] e CN (Contention Nodes) [23] consideram a interferência interfluxo no cálculo de suas rotas. A métrica MIC utiliza o componente IRU (Interference-aware Resource Usage) para calcular a interferência interfluxo. A utilização do IRU faz com que a métrica MIC possa calcular em uma transmissão o tempo que seria consumido nos canais de seus vizinhos. Dessa forma as rotas mais procuradas serão aquelas que ocupam menos tempo nos canais de seus vizinhos. $\mathrm{Na}$ métrica $\mathrm{CN}$, quando um nó recebe ou envia dados para seus vizinhos automaticamente ele é classificado. Nessa classificação destacam-se os nós que estão enviando dados e os nós que estão recebendo dados. A métrica $\mathrm{CN}$ pode calcular as melhores rotas levando em conta o número de vizinhos que estão enviando dados e possivelmente causando interferência com suas transmissões.

Vale ressaltar que a maior parte dos protocolos de roteamento implementa a métrica ETX ou pequenas variações desta [8].

\section{A MÉTRICA ALTERNATIVE PATH (AP)}

A métrica AP, sendo proposta neste trabalho, é baseada na métrica ETX, porém diferencia-se dela por levar em conta o número de nós vizinhos nos cálculos de qualidade de enlaces.
A interferência causada por nós vizinhos em uma rota pode comprometer significativamente o desempenho da rede.

No ETX, o cálculo de qualidade de um enlace é feito usando o inverso da multiplicação da qualidade do Link Direto, LD, pela qualidade do Link Reverso, LR (Equação 1):

$$
\mathrm{ETX}=1 /(\mathrm{LD} \times \mathrm{LR})
$$

É importante notar que a métrica ETX calcula seu LR através do número de pacotes recebidos em um intervalo de tempo. Para isso cada nó envia, periodicamente, uma mensagem-sonda (probe) com informações para a manutenção das rotas. Por exemplo, considere-se um intervalo de $20 \mathrm{~s}$ e a transmissão da mensagem sonda a cada $2 \mathrm{~s}$ de cada nó para seus vizinhos. Sendo assim, um enlace de qualidade $100 \%$ teria recebido 10 pacotes em um intervalo de 20 s. O cálculo do LD é feito da mesma forma, porém é informado pelos vizinhos quando os mesmos adicionam essa informação à mensagemsonda; ou seja, cada nó indica na mensagem-sonda o número de pacotes recebidos de cada um de seus vizinhos.

$\mathrm{O}$ cálculo da métrica AP modifica o cálculo original do ETX, adicionando ao fator $L D$ da Equação 1 o valor de $Y$. Esse valor é justamente a divisão de um peso $(P)$ pelo número de vizinhos $(N V)$ do nó, de acordo com as Equações 2 e 3:

$$
\mathrm{Y}=\mathrm{P} / \mathrm{NV} \text {. }
$$

\section{Portanto}

$$
\mathrm{AP}=1 /((\mathrm{LD}+\mathrm{Y}) \times \mathrm{LR})
$$

Como $N V$ representa o número de vizinhos, quanto maior a quantidade de vizinhos menor será a contribuição de $Y$ para o cálculo da qualidade de um enlace. Ou seja, em um enlace com um número muito alto de vizinhos o valor de $Y$ seria de pouca influência, porém em um enlace com poucos vizinhos, $Y$ teria uma influência significativa (maior probabilidade do enlace de ser o escolhido). Assim, a métrica AP procura evitar perdas causadas por interferência interfluxo de outros nós, vizinhos. Para isso, rotas menos suscetíveis a interferências são selecionadas, melhorando o desempenho da rede.

Rota A, NV=15

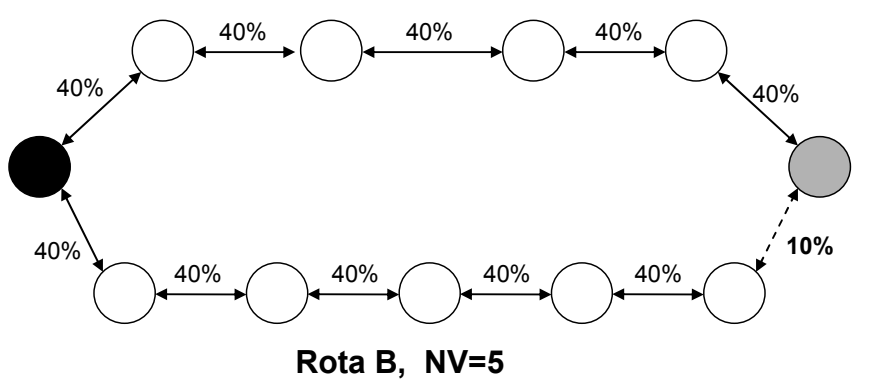

Fig 1. Exemplo de cálculo de rotas.

Para ilustrar o cálculo da métrica AP considere o exemplo da Figura 1, que apresenta 2 rotas, $A$ e $B$, para um mesmo destino. A rota $A$ possui um total de 5 enlaces com $40 \%$ de probabilidade de perda em cada enlace. A rota $B$ tem 6 enlaces sendo que 5 com probabilidade de perda de $40 \%$ e 1 com 
probabilidade de perda de $10 \%$. Tal cenário, recorrente em redes em malha, se justifica, por exemplo, se a rota $A$ passa por regiões com muitos nós vizinhos (não mostrados na figura), em que cada nó da rota tenha, digamos, 15 vizinhos. A rota $B$, por sua vez, passa por um caminho mais longo e mais afastado dos outros nós (não mostrados na figura). Neste caso cada nó possui apenas 5 vizinhos.

Com base nos números de vizinhos por nó e considerando um peso $P$ de 0,6 , o valor de $Y$ para cada nó na rota $A$ vale 0,04 e na rota $B, Y$ vale 0,12 . Os valores $L D$ e LR podem ser calculados através da probabilidade de perda. Assim, um enlace com $40 \%$ de probabilidade de perda teria uma probabilidade de entrega ou qualidade de enlace de $60 \%(0,6)$. Já um enlace com $10 \%$ de perda teria uma probabilidade de entrega ou qualidade de enlace de $90 \%(0,9)$.

$\mathrm{O}$ custo total de cada rota seria calculado pelo somatório de todos os custos dos $A P \mathrm{~s}$ intermediários através da Equação 3. Para a rota $A$, obtém-se o valor 2,6 para cada enlace, como são 5 enlaces com a mesma média de vizinhos e probabilidade de perda, o somatório do custo da rota é 13. Para o custo total da rota $B$ seriam calculados os custos dos $A P$ s de 5 enlaces com perda de $40 \%$ e o de um enlace com perda de $10 \%$. Somando os custos dos APs dos 5 enlaces com $40 \%$ de perda tem-se 11,55 e somando-se a este o custo do $A P$ do enlace de $10 \%$ de perda, que é de 1,09, tem-se um custo total da rota de 12,64. Portanto utilizando a métrica AP, a rota $A$ teria um custo total de 13 e a rota $B$ teria um custo de 12,64 . Como a heurística da métrica AP escolhe rotas com menor custo, a rota $B$ seria a escolhida, mesmo tendo um caminho mais longo, pelo fato desta apresentar uma menor probabilidade de perda por interferência interfluxo.

Utilizando a métrica ETX neste cenário os custos de cada rota mudariam, já que o ETX não levaria em conta a interferência interfluxo e, portanto, não consideraria o valor $Y$ na sua equação. Assim, a rota $A$ utilizando o ETX teria um custo igual à soma dos 5 enlaces com $40 \%$ de perda, obtendo um custo total de 13,9 . Para a rota $B$, seria utilizada a mesma soma dos 5 enlaces com perda de $40 \%$ adicionada ao custo do enlace com $10 \%$ de perda, obtendo um custo total de 15,13 . Como a rota $A$ teria um custo total mais baixo, a mesma seria a escolhida pela métrica ETX; ou seja, neste caso a rota utilizada seria justamente aquela com maior probabilidade de sofrer interferências interfluxo.

\section{SimulaçõES}

Para avaliar o comportamento da métrica AP, realizamos uma série de simulações utilizando a ferramenta $n s-2$ [24]. Foram também utilizadas extensões para o OLSR e a métrica ETX desenvolvidas para o $n s-2$ [25]. O protocolo de roteamento OLSR foi utilizado para implantar a métrica AP, pois este já faz o cálculo do número de nós vizinhos para selecionar, a partir do mesmo, o conjunto de nós MPR (Seção 2). Essa característica nata do OLSR facilita a implementação da métrica AP, pois a informação contendo o número de vizinhos já está disponível na estrutura deste protocolo. Sobre este arcabouço implementamos adaptações para contemplar os cálculos da métrica AP.
Selecionamos um cenário (Figura 1) contendo algumas rotas com maior interferência interfluxo e outras com baixa interferência. As linhas tracejadas indicam enlaces com perdas superiores a $50 \%$, já as linhas contínuas indicam enlaces com melhor qualidade. Foram escolhidos alguns nós $(3,5,7,9,15$, 17 em cor cinza) para receber fluxos CBR, provenientes do nó origem (nó 0), por um tempo fixo. Estes "nós-termômetro" foram utilizados para estudar o comportamento da rede.

Nas simulações, as mensagens de sonda, HELLO no OLSR, foram enviadas a cada $2 \mathrm{~s}$. As mensagens de estado do enlace, utilizadas pelos nós MPRs, são enviadas a cada $5 \mathrm{~s}$. A taxa de perdas no meio é calculada utilizando como base 10 amostras de transmissões de HELLO. As transmissões CBR entre o nó origem e os nós selecionados foram feitas em um total de $45 \mathrm{~s}$ a uma taxa de $12 \mathrm{kbps}$ para cada nó selecionado..Os outros nós da rede foram submetidos a cargas variadas de transmissão com fontes e destinos aleatórios e com taxas variando entre 6 e $12 \mathrm{kbps}$. As transmissões para os nós selecionados foram mantidas em todas as simulações, enquanto as outras conexões, de background, variaram em número e duração. Assim formouse um cenário com múltiplas interferências e com transmissões concorrentes. Foram utilizados nas simulações alguns parâmetros como: Path Loss Exponent com valor igual a 2,7 e Shadowing Deviation com o valor igual a 4 . Tais valores também foram utilizados em [25] para simular um ambiente de uma rede em malha sem fio real. Estes parâmetros representam algumas particularidades das redes reais como alta incidência solar, o elevado índice pluviométrico e a concentração de obstáculos nas proximidades da rede.

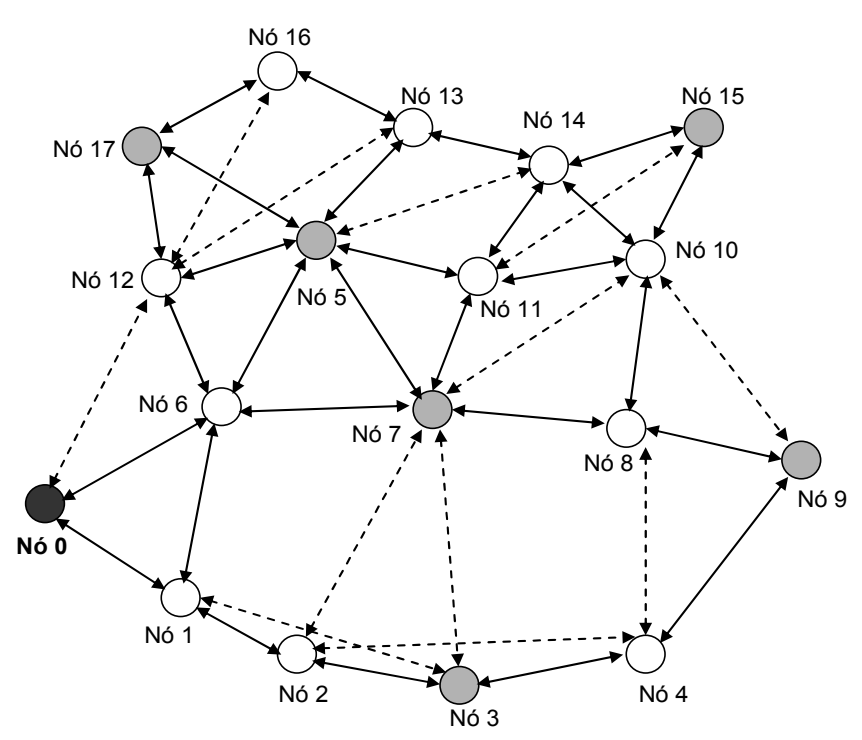

Fig 2. Rede utilizada nas simulações.

Foram medidos, com intervalo de confiança de $90 \%$, os valores médios da taxa de perda, do atraso, da vazão e do tamanho da rota.

As simulações com a métrica AP utilizando peso $(P)$ igual a 0,6 foram comparadas com a métrica ETX. O valor de $P$ foi escolhido em função de simulações preliminares realizadas com a métrica AP para diversos valores de $P$. Os pesos com 
valores próximos de 0,6 , como 0,5 e 0,7 , apresentaram resultados similares porém inferiores aos obtidos com o peso 0,6 . Reduzindo ou aumentando os pesos, como nos casos de $P$ igual a 0,1 e 1 , a métrica começa a ter um desempenho inferior para alguns enlaces. Isso se deve ao fato de que ao se aumentar muito o peso, a métrica começa a forçar uma grade parte do tráfego para os caminhos periféricos e ao se reduzir muito o peso, a métrica começa a se comportar de forma semelhante à métrica ETX.

\section{A. Resultados}

Nas Figuras 3 a 6, são apresentados os resultados das simulações para os seis nós(-termômetro) selecionados. Estes foram dispostos no eixo $x$ segundo suas distâncias e possibilidades de rotas alternativas em relação ao nó 0 , de forma a evidenciar a atuação da métrica AP em comparação com a ETX.

A Figura 3 apresenta a taxa média de perda de pacotes. Pode ser observado que a métrica AP obteve menores perdas. Sua taxa de perdas apenas se aproximou da métrica ETX para o nó destino 17, que apresenta poucas chances de se evitar a interferência interfluxo, devido às pequenas possibilidades de rotas existentes (vide topologia na Figura 2).

A Figura 4 mostra que a métrica AP atingiu um menor atraso médio para 5 dos 6 destinatários. A rota para o nó 9 apresentou maior diferença entre as métricas AP e ETX por possuir caminhos longos e com uma boa possibilidade de escolha de rotas alternativas com pequena interferência interfluxo.

A Figura 5 mostra que a métrica AP proporcionou vazão média superior todos os nós. A rota até o nó 17 , além de ter apresentado uma taxa de perda aproximada entre as duas métricas, também apresentou uma taxa de vazão aproximada. Este comportamento comprova o que foi observado no gráfico de perdas, em relação às pequenas possibilidades de escolha de rotas alternativas.

A Figura 6 apresenta o tamanho médio da rota em número de saltos (hops). Observa-se que a métrica AP utilizou rotas mais longas em relação às rotas usadas pela métrica ETX. Essa diferença do tamanho de rotas se mostrou maior principalmente nas rotas mais longas como é o caso dos nós destinatários 9 e 15, pois a métrica AP procurou caminhos alternativos, eventualmente com mais saltos, para poder evitar os danos causados pela interferência interfluxo em suas rotas.

Para as simulações realizadas, verifica-se que a métrica AP apresenta desempenho médio $6,5 \%$ melhor em relação às perdas, 6,7\% melhor em relação à vazão e 10,6\% melhor em relação ao atraso. Como esperado, a métrica AP utilizou em média caminhos mais longos para proporcionar um melhor desempenho da rede.

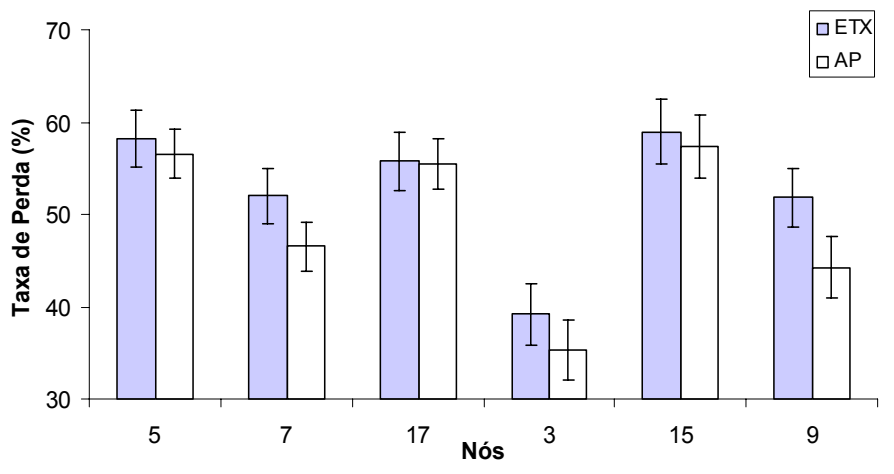

Fig 3. Taxa média de perda de pacotes.

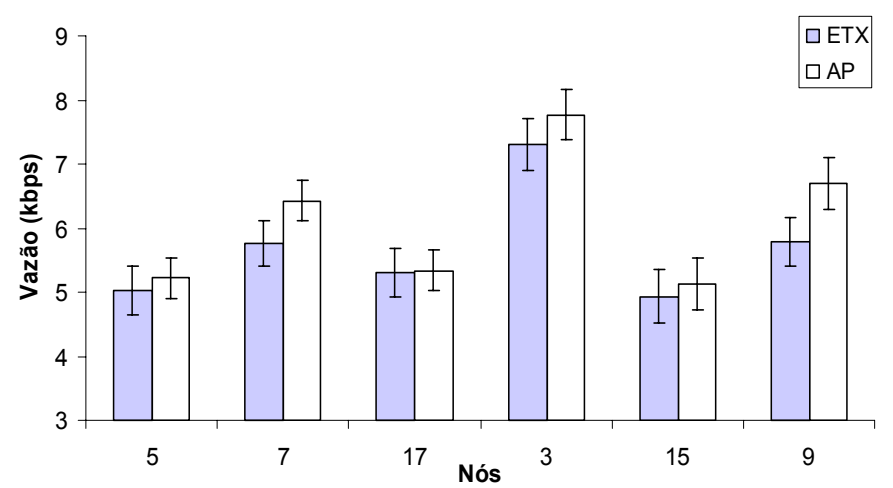

Fig 5. Vazão média.

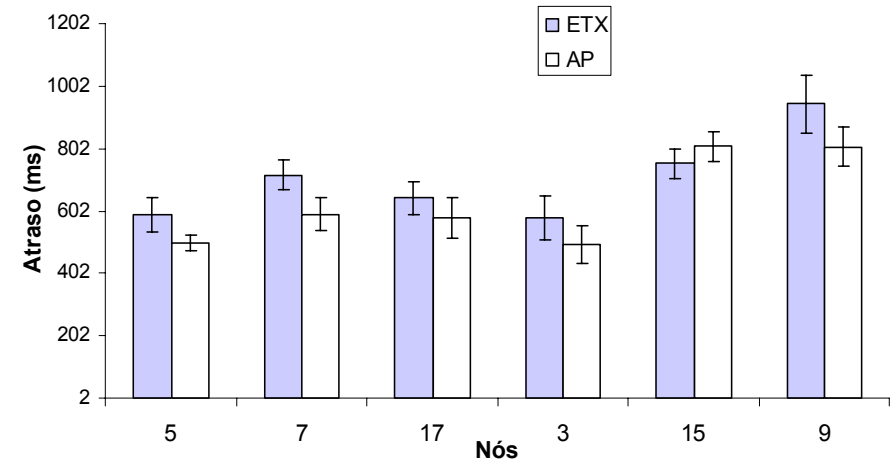

Fig 4. Atraso médio.

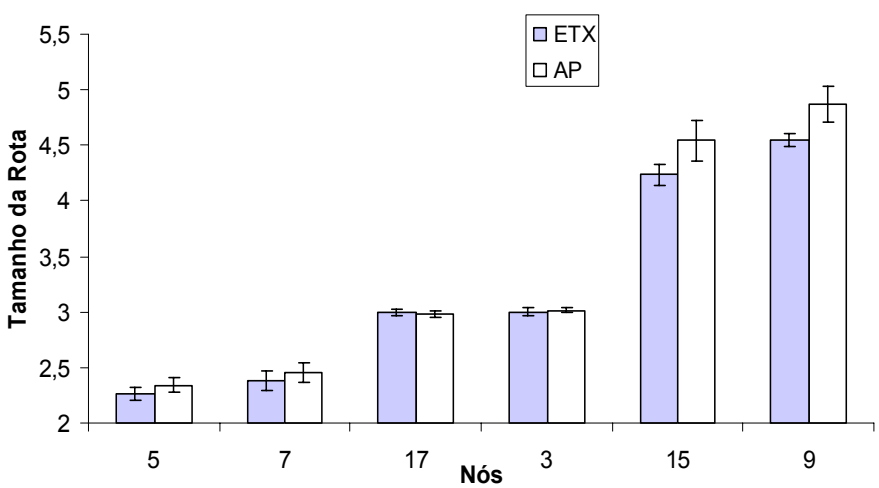

Fig 6. Tamanho médio da rota. 


\section{CONCLusÕES}

Este trabalho propôs uma nova métrica a AP para aprimorar o cálculo de rotas em redes em malha, que considera a probabilidade de interferência dos nós vizinhos nas transmissões de outras rotas. A métrica AP utiliza como base o cálculo de probabilidade de transmissões feito pela métrica ETX, podendo por isso ser facilmente incorporada a infraestruturas existentes.

Nas simulações realizadas a métrica AP apresentou melhores resultados com relação a perda de pacotes, vazão e atraso em relação ao ETX. Este desempenho superior se deve ao fato da métrica AP procurar caminhos alternativos aos caminhos com grande probabilidade de interferências por nós vizinhos.

$\mathrm{Na}$ seqüência das pesquisas será estudado o comportamento da métrica AP em redes maiores. Pretende-se testar a métrica em cenários com mais interferências por nós vizinhos e com mais rotas alternativas.

\section{AGRADECIMENTOS}

Dalbert Mascarenhas é bolsista CAPES. Os autores agradecem o apoio parcial da Faperj e CNPq.

\section{REFERÊNCIAS}

[1] S. Kim, S. Lee e S. Choi, "The Impact of IEEE 802.11 MAC Strategies on Multi-Hop Wireless Mesh Networks", IEEE Workshop on Wireless Mesh Networks, setembro de 2006.

[2] D. Passos, D. Teixeira, D.C. Muchaluat-Saade, L.C. Schara Magalhães e C. Albuquerque, "Mesh Network Performance Measurements", $5^{\text {th }}$ International Information and Telecommunications Technologies Symposium, dezembro de 2006.

[3] J. Bicket, D. Aguayo, S. Biswas e R. Morris, "Architecture and Evaluation of an Unplanned 802.11b Mesh Network", ACM MobiCom, p. 31-42, agosto de 2005.

[4] R. Bruno, M. Conti e E. Gregori, "Mesh Networks: Commodity Multihop Ad Hoc Networks", IEEE Communications Magazine, vol 43, p. 123131, março de 2005 .

[5] CUWin, http://www.cuwireless.net, acessado em abril de 2008.

[6] I. Akyildiz, X. Wang e W. Wang, "Wireless Mesh Networks: A Survey", Elsevier, p. 445-487, 2005.

[7] Y. Yang, J. Wang e R. Kravets, "Designing Routing Metrics for Mesh Networks", IEEE Workshop on Wireless Mesh Networks, setembro de 2005 .
[8] D. Passos e C. Albuquerque, "Proposta, Implementação e Análise de uma Métrica de Roteamento Multiplicativa para Redes em Malha Sem Fio", Revista Eletrônica de Iniciação Científica (REIC), setembro de 2007.

[9] C. E. Perkins, "Ad Hoc On-Demand Distance Vector (AODV) Routing", RFC 3561, julho de 2003.

[10] D. Johnson, D. Maltz e J. Broch, "DSR: The Dynamic Source Routing Protocol for Multihop Wireless Ad Hoc Networks", Ad Hoc Networking, Addison-Wesley Longman Publishing Co., Boston, MA, 2001.

[11] R. Draves, J. Padhye e B. Zill, "Comparison of Routing Metrics for Static Multi-hop Wireless Networks”. Em ACM MobiCom, p. 114-128, 2004.

[12] D. Aguayo, J. Bicket e R. Morris, "SrcRR: A High Throughput Routing Protocol for 802.11 Mesh Networks" (DRAFT). MIT, 2005.

[13] R. Draves, J. Padhye e B. Zill, "Routing in Multi-radio, Multi-hop Wireless Mesh Networks", Em ACM MobiCom, p. 114-128, 2004.

[14] M. E. Campista, L. H. Costa e O. C. Duarte, "Um Algoritmo Eficiente de Disseminação dos Estados de Enlace para Redes em Malha Sem Fio", $25^{\circ}$ Simpósio Brasileiro de Redes de Computadores e Sistemas Distribuídos, 2007.

[15] G. Pei, M. Gerla e T. W. Chen, "Fisheye state routing in mobile ad hoc networks", ICDCS Workshop on Wireless Networks and Mobile Computing, p. D71-D78, 2000

[16] MITRE Corporation, "Mobile mesh routing protocol", http://www.mitre.org/work/tech transfer/ mobilemesh/draft-grace-manetmmrp-00.txt, 2006.

[17] OLSR Implementation, http://www.olsr.org, acessado em abril de 2008

[18] C. Santivanez et al., "On the Scalability of Ad Hoc Routing Protocols", IEEE INFOCOM, vol. 3, p. 1688-1697, junho de 2002.

[19] D. Couto, D. Aguayo, J. Bicket e R. Morris, "A High-Throughput Path Metric for Multi-Hop Wireless Routing”, ACM MobiCom, setembro de 2003.

[20] D. C. Muchaluat-Saade, C. Albuquerque, L.C. Schara Magalhães, D. Passos, J. Duarte, R. Valle, "Redes em Malha: Solução de Baixo Custo para Popularização do Acesso à Internet no Brasil", XXV Simpósio Brasileiro de Telecomunicações, SBrT, setembro de 2007.

[21] T. Salonidis, M. Garetto, A. Saha e E. Knightly, "Identifying High Throughput Paths in 802.11 Mesh Networks: a Model-based Approach", IEEE International Conference, p. 21-30, outubro de 2007.

[22] Y. Yang, J. Wang e R. Kravets, "Interference-aware Load Balancing for Multihop Wireless Networks", UIUCDCS-R-2005-2526, 2005.

[23] B. C. Kim, "Performance Comparison of Route Metrics for Wireless Mesh Networks", IEICE-Transactions on Communications, vol. E89-B, p. 3124-3127, novembro de 2006.

[24] Network simulator ns-2, http://www.isi.edu/nsnam/ns/, acessado em abril de 2008.

[25] W. Cordeiro, E. Aguiar, W. Moreira, A. Abelém e M. Stanton, "Providing Quality of Service for Mesh Networks Using Link Delay Measurements", Proceedings of 16th International Conference on Computer Communications and Networks, p. 991-996, agosto de 2007. 